Revista Brasileira do Esporte Coletivo - v. 1, n,2, 2017.

Artigo original

\section{AÇÕES DE LEVANTAMENTO: INDICADORES DE RENDIMENTO NO VOLEIBOL ESCOLAR}

\author{
Yago Pesoa da Costa'; Jakenio Cavalcante \\ Batista $^{2}$; Paloma de Araújo Cruz ${ }^{3}$; Gilmário \\ Ricarte Batiste 4 .
}

Título resumido:

Indicadores de rendimento no voleibol

\title{
RESUMO
}

Introdução: Diante das diversas funções de jogo possíveis no voleibol, o levantador destaca-se, visto que é responsável pela distribuição das bolas para os atacantes. Objetivo: O objetivo foi associar a qualidade das ações do levantamento com a zona de distribuição, e de forma complementar comparar a qualidade entre sets vencidos e perdidos. Método: O estudo teve a participação de 12 instituições de ensino, que competiram durante os Jogos Escolares da Juventude 2016. Foram observados 32 sets, pertencentes a 16 jogos da competição, contabilizando 1656 ações de levantamento. Para verificar a associação foi utilizado o teste do qui-quadrado, e para comparar o teste U de Mann-Whitney. Resultados: Foram obtidos os seguintes resultados: A distribuição para a zona ofensiva teve maior incidência $(91,1 \%)$. Houve associação significativa da zona de distribuição com a qualidade do levantamento $\left(x^{2}=51.810 ; p<0,001\right)$. Ao realizar o levantamento para a zona ofensiva, a frequência de ações excelentes foi maior do que o esperado. Já o set perdido apresentou maior número de levantamentos que não geram opções de ataque $(U=362,00 ; p=0,034)$. Quanto ao coeficiente de performance não foi encontrado diferença entre vencidos e perdidos. Conclusão: Concluímos que a qualidade do levantamento apresentou uma dependência com as zonas ofensivas e defensivas, e os sets perdidos foram superiores em levantamentos que não geram ataque, o que favorece ao contra-ataque do set vencido.

Palavras-chave: Eficiência, destreza motora, atletas, adolescente.

\section{ABSTRACT}

Introduction: Given the various possible game functions in volleyball, the lifter stands out as he is responsible for distributing the balls to the attackers. Objective: The objective was to associate the quality of the actions of the survey with the distribution zone, and in a complementary way to compare the quality between overdue and lost sets. Method: The study had the participation of 12 educational institutions that competed during the 2016 Youth School Games. There were observed 32 sets, belonging to 16 games of the competition, counting 1656 actions of survey. To verify the association, the chi-square test was used, and to compare the Mann-Whitney U-test. Results: The following results were obtained: Distribution to the offensive zone had a higher incidence $(91.1 \%)$. There was a significant association between the distribution zone and the quality of the survey $(x 2=51.810 ; p$ $<0.001)$. When conducting the survey for the offensive zone, the frequency of excellent actions was higher than expected. On the other hand, the lost set had the highest number of surveys that did not generate attack options $(U=362.00, p=0.034)$. Regarding the performance coefficient, no difference was found between losers and losers. Conclusion: We conclude that the quality of the survey showed a dependence on the offensive and defensive zones, and the lost sets were superior in surveys that do not generate an attack, which favors the counterattack of the defeated set.

Key words: Efficiency, motor skills, athletes, adolescent.

1234 Universidade Federal da Paríba, João Pessoa, Paraíba - Brasil. e-mail: yago_pessoa@hotmail.com 
INDICADORES DE RENDIMENTO NO VOLEIBOL ESCOLAR

INTRODUÇÃO

O voleibol desenvolve-se a partir de duas estruturas básicas denominadas por complexos, sendo o primeiro composto pelas ações da recepção de saque, levantamento e ataque (organização da fase ofensiva) e o segundo pelo bloqueio, defesa e contra-ataque (organização da fase defensiva) (PALAO, SANTOS, UREÑA, 2004). Além disso, existem outras abordagens que ampliam esses complexos em mais segmentos, caracterizando a continuidade subsequente do jogo (HILENO, BUSCÀ, 2012; PALAO, MANZANARES, ORTEGA, 2015). Diante dessas estruturas, os atletas que compõem uma equipe podem assumir diferentes funções no jogo, como ponteiro, meio de rede, oposto, líbero e levantador para desempenhar as ações técnico-táticas determinantes a vitória do set (MILIĆ et al., 2017; COSTA et al. 2017).

A ligação entre as ações dá ao jogo uma característica cíclica, como uma lógica bem definida (MARCELINO et al., 2010). No entanto, as ações denominadas de terminal, caracterizadas pela conquista do ponto, parecem ser determinantes ao sucesso do jogo (COSTA et al., 2014). Em um estudo durante a Liga Mundial $2005 \mathrm{com}$ atletas masculinos, observou-se que 0 ataque e o bloqueio foram decisivos na vitória, e o saque associou-se ao ranking final da competição (MARCELINO, MESQUITA, AFONSO, 2008). No caso específico do saque, o mesmo pode desestabilizar a organização ofensiva dos adversários, sobretudo em equipes de formação femininas prejudicando a recepção de saque (FERNANDEZ-ECHEVERRIA et al., 2015) e consequentemente o levantamento.

Assim sendo, pelo fato da ação terminal ser determinante no jogo, os jogadores que atuam na função de atacante desempenham importante papel, já que são essas ações que conquistam mais pontos durante uma partida (VALLADARES, GARCÍA-TORMO, JOÃO, 2016). Porém, esse tipo de ação dependente da qualidade da recepção de saque (COSTA et al., 2011), e de forma subsequente do levantamento que são ações de continuidade, ou seja, promovem excelência na sequência do jogo (PALAO, MANZANARES, ORTEGA, 2009). Para Matias e Greco (2011a), o levantador atua como um jogador chave durante o jogo, percebendo a equipe adversária (bloqueadores), sua própria equipe (atacantes disponíveis) e diante da ação antecedente, tomar a decisão da direção e da velocidade do levantamento (MATIAS, GRECO, 2011b).

Portanto, parece ser uma questão importante investigar o levantamento pela sua contribuição na organização do jogo. Neste sentido, Ramos et al., (2004) investigaram a incidência da distribuição, condições de levantamento, circunstâncias de finalização subsequente e os seus efeitos, em equipes masculinas durante a fase final da Superliga 2002/2003 (Campeonato Brasileiro de Clubes). Posteriormente, Matias e Greco, (2011b) realizaram estudo semelhante, sendo que a amostra contou com os levantadores campeões de ambos os naipes. Além disso, outros estudos investigaram 0 conhecimento táticoestratégico dos levantadores devido à importância de suas tomadas de decisões (MATIAS; GRECO, 2011c; QUEIROGA et al., 2010).

Contudo, percebe-se certa incipiência de indicadores de performance no que concerne à ação do levantamento nas categorias de formação de jovens atletas, visto que essa atividade é fundamental para a organização ofensiva do jogo. Diante disso, o objetivo desta pesquisa foi associar a qualidade das ações do levantamento com a zona de distribuição, e de forma complementar comparar a qualidade entre sets vencidos e perdidos.

\section{MATERIAIS E MÉTODOS}

O presente estudo contou com a participação de 12 instituições educacionais, representante dos Estados de Alagoas, Pará, Santa Catarina, Amazonas, Rio de Janeiro, Ceará, São Paulo, Rio Grande do Norte, Minas Gerais, Rio Grande do Sul, Maranhão e Paraíba, que competiram durante os Jogos Escolares da Juventude 2016 (correspondente ao Campeonato Brasileiro Escolar), realizado em João Pessoa - PB, divisão especial, naipe feminino, categoria 15-17 anos. Foram avaliados 16 jogos (32 sets, sendo excluídos os sets tie break), correspondente a 1656 levantamentos por atletas especialistas nesta função. Os dados foram agrupados em relação à zona de distribuição e em sets vencidos e perdidos.

Todos os atletas que aceitaram participar voluntariamente assinaram 0 termo de assentimento e tiveram sua participação autorizada pelo responsável legal por meio do termo de consentimento livre e esclarecido, ambos elaborados de acordo com a declaração de Helsinki. Previamente à coleta de dados, todos os procedimentos foram aprovados junto ao Comitê de Ética em Pesquisa com Seres Humanos da Universidade Federal da Paraíba, locada no Centro de Ciências da Saúde (UFPB-CCS), protocolo $441 / 14$.

\section{PROCEDIMENTOS}

O levantamento de toque foi avaliado pela qualidade da ação em relação ao ataque, sendo utilizada uma escala de quatro pontos: $0=$ erro, $1=$ sem opções de ataque, $2=$ ataque com opções limitadas e $3=$ todas as opções de ataque, conforme utilizado em estudo anterior (PALAO, MANZANARES, ORTEGA, 2009). Para efeito do estudo foi realizada uma adaptação nas zonas de distribuição (posição para onde o levantamento foi realizado), sendo agrupadas em zona ofensiva (2, 3 e 4) e defensiva (1, 6 e 5) (AFONSO et al., 2012; MAIA, MESQUITA, 2006), conforme figura 1.

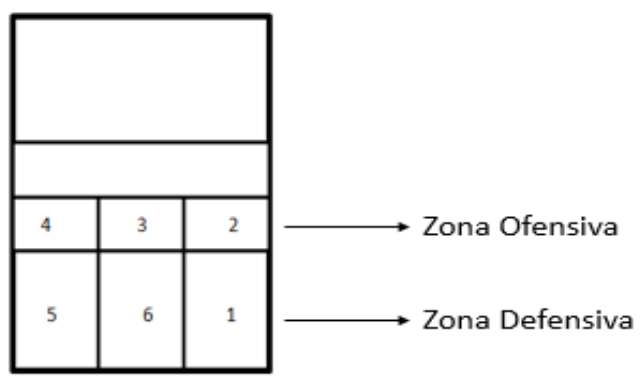

A coleta de dados foi realizada em duas etapas. A primeira consistiu-se em filmar todos os jogos utilizando uma câmera filmadora (Samsung ${ }^{\circledR}$ HMX-F90), apoiada em tripé, a uma distância aproximada de 10 metros, em plano elevado a 04 metros de altura. Em seguida, os jogos foram analisados em um notebook (Samsung ${ }^{\circledR}$ ATIV Book 2 270E4E-KD5, tela de 14 polegadas, com sistema operacional Windows $10^{\circledR}$ - Fabricado no Brasil, 
2013), tendo os dados anotados no software Microsoft Excel ${ }^{\circledR}$ 2016. Para garantir confiabilidade nas avaliações foram realizados procedimentos intra e inter, com dois avaliadores. A primeira intra foi realizada com 15 dias (JAMES, TAYLOR, STANLEY, 2007), e, em seguida, foi realizada a avaliação inter, ambas por meio do teste de Kappa, obtendo valores superiores a 0,80 , o que permitiu a continuidade das avaliações seguintes (LANDIS, $\mathrm{KOCH}, 1977)$.

A partir da escala foi calculado o coeficiente utilizando a equação Coeficiente de Performance de levantamento= $1,5^{*}$ (ações do tipo "1" e "2") + $3^{*}$ (ações tipo "3") / Soma de todas as ações, conforme proposta de Coleman (2002).

\section{ANÁLISE ESTATIISTICA}

A estatística descritiva foi utilizada por meio de distribuição de frequência (absoluta e relativa), média, mediana, desvio padrão e intervalo interquartil (25-75). Foi utilizado o teste de Quiquadrado com correção de Monte Carlo para verificar a associação entre o levantamento e a zona de distribuição. Quando encontradas diferenças, foram utilizados os resíduos ajustados, assim, foi observado o ponto de maior ou menor frequência em relação ao esperado, levando-se como referência os valores de resíduos positivos maior que 2 e negativo menor que -2 (Costa et al., 2011). Para as comparações entre sets vencidos e perdidos foi utilizado o teste de $U$ de Mann-Whitney. O nível de significância estabelecido para todas as análises foi de $5 \%$. O software IBM ${ }^{\circledR}$ SPSS $^{\circledR}(20.0 .0$, 2011) foi usado para realizar todos os cálculos estatísticos.

\section{RESULTADOS}

Em relação à zona de distribuição, houve maior frequência de levantamento para a zona ofensiva $(91,1 \%)$. Além disso, houve associação significativa entre a zona de distribuição e a qualidade do levantamento $\left(x^{2}=51.810 ; p<0,001\right)$. Quando o levantamento foi distribuído para a zona ofensiva, houve frequência maior do que o esperado $(5,9)$ de ações com o escore 3 , de maneira oposta, ações de levantamento para a zona defensiva apresentaram frequência menor que a esperada ($5,9)$ na qualidade do levantamento (Tabela 1).

Tabela 1. Associação entre a zona de distribuição e a qualidade do levantamento.

\begin{tabular}{|c|c|c|c|c|c|c|}
\hline \multirow{2}{*}{$\begin{array}{c}\text { Zona de } \\
\text { distribuição }\end{array}$} & & \multicolumn{4}{|c|}{ Escores } & \multirow[b]{2}{*}{ Total } \\
\hline & & 0 & 1 & 2 & 3 & \\
\hline \multirow{5}{*}{1} & Frequência & 45 & 105 & 205 & 1154 & 1509 \\
\hline & $\begin{array}{l}\text { Frequência } \\
\text { esperada }\end{array}$ & 41 & 120,3 & 223,3 & 1124,5 & 1509 \\
\hline & $\begin{array}{c}\% \text { zona de } \\
\text { levantamento }\end{array}$ & $3,0 \%$ & $7,0 \%$ & $13,6 \%$ & $76,5 \%$ & $100,00 \%$ \\
\hline & $\begin{array}{l}\text { \% escore } \\
\text { observado }\end{array}$ & $100,00 \%$ & $79,5 \%$ & $83,7 \%$ & $93,5 \%$ & $91,1 \%$ \\
\hline & $\begin{array}{l}\text { Resíduos } \\
\text { ajustados }\end{array}$ & 2,1 & $-4,9$ & $-4,4$ & 5,9 & \\
\hline \multirow{5}{*}{2} & Frequência & $<5$ & 27 & 40 & 80 & 147 \\
\hline & $\begin{array}{l}\text { Frequência } \\
\text { esperada }\end{array}$ & 4 & 11,7 & 21,7 & 109,5 & 147,0 \\
\hline & $\begin{array}{c}\% \text { zona de } \\
\text { levantamento }\end{array}$ & $\mathrm{n}<5$ & $18,4 \%$ & $27,2 \%$ & $54,4 \%$ & $100,0 \%$ \\
\hline & $\begin{array}{l}\text { \% escore } \\
\text { observado }\end{array}$ & $\mathrm{n}<5$ & $20,5 \%$ & $16,3 \%$ & $6,5 \%$ & $8,9 \%$ \\
\hline & $\begin{array}{l}\text { Resíduos } \\
\text { ajustados }\end{array}$ & $\mathrm{n}<5$ & 4,9 & 4,4 & $-5,9$ & \\
\hline \multirow{4}{*}{ Total } & Frequência & 45 & 132 & 245 & 1234 & 1656 \\
\hline & $\begin{array}{c}\text { Frequência } \\
\text { esperada }\end{array}$ & 45 & 132 & 245 & 1234 & 1656 \\
\hline & $\begin{array}{c}\% \text { zona de } \\
\text { levantamento }\end{array}$ & $2,7 \%$ & $8,0 \%$ & $14,8 \%$ & $74,5 \%$ & $100 \%$ \\
\hline & $\begin{array}{l}\% \text { escore } \\
\text { observado }\end{array}$ & $100,0 \%$ & $100 \%$ & $100 \%$ & $100 \%$ & $100 \%$ \\
\hline
\end{tabular}

1= zona ofensiva (zonas 2, 3 e 4); $2=$ zona defensiva (zonas 1, 5 e 6). $0=$ erro, $1=$ sem opções de ataque, $2=$ ataque com opções limitadas e $3=$ todas as opções de ataque. $x^{2}=51.810 ; p<0,001$.

O levantamento pareceu ser bastante semelhante quando comparado entre sets vencidos e perdidos. A exceção decorreu-se nas ações do tipo 1, que apresentou diferença significativa $(p=0,034)$ (Tabela 2). $O$ coeficiente de performance seguiu o conjunto, e não apresentou diferença significativa $(p=0,330)$. 
Tabela 2. Comparação da contagem dos escores do levantamento e coeficiente de performance entre sets vencidos e perdidos em atletas escolares.

\begin{tabular}{|c|c|c|c|c|c|c|c|}
\hline Escore & Resultado do Set & Mediana & $\begin{array}{c}\text { IIQ } \\
(25-75 \%)\end{array}$ & Média & DP & U & $p$ \\
\hline \multirow{2}{*}{0} & Vencidos & 0 & $0-1$ & 0,56 & 0,759 & \multirow{2}{*}{491,50} & \multirow{2}{*}{0,757} \\
\hline & Perdidos & 0 & $0-1$ & 0,59 & 0,712 & & \\
\hline \multirow{2}{*}{1} & Vencidos & 1 & $2-1,75$ & 1,34 & 1,15 & \multirow{2}{*}{362,00} & \multirow{2}{*}{0,034} \\
\hline & Perdidos & 2 & $1-3$ & 2,03 & 1,42 & & \\
\hline \multirow{2}{*}{2} & Vencidos & 2 & $2-4$ & 2,72 & 1,15 & \multirow{2}{*}{426,50} & \multirow{2}{*}{0,243} \\
\hline & Perdidos & 3 & $2-4,75$ & 3,22 & 1,77 & & \\
\hline \multirow{2}{*}{3} & Vencidos & 15 & $11-18,75$ & 15,06 & 5,43 & \multirow{2}{*}{497,00} & \multirow{2}{*}{0,804} \\
\hline & Perdidos & 14,50 & $13-17$ & 15,22 & 4,63 & & \\
\hline \multirow[t]{2}{*}{ CPL } & Vencidos & 2,61 & $2,45-2,73$ & 2,58 & 0,20 & \multirow{2}{*}{439,50} & \multirow{2}{*}{0,330} \\
\hline & Perdidos & 2,59 & $2,40-2,70$ & 2,53 & 0,20 & & \\
\hline
\end{tabular}

*Diferença significativa; $\mathrm{CPL}=$ Coeficiente de performance de levantamento; $\mathrm{IIQ}=$ Intervalo interquartil. $\mathrm{DP}=$ Desvio Padrão. $0=$ erro; $1=$ sem opções de ataque; $2=$ ataque com opções limitadas; e $3=$ todas as opções de ataque.

Na porcentagem das ações por set também não foi encontrado diferença significativa entre as ações, porém houve tendência nas ações de tipo 1, similar à contagem por set (Tabela 3).

Tabela 3. Comparação das porcentagens das ações de levantamento entre sets vencidos e perdidos.

\begin{tabular}{|c|c|c|c|c|c|c|c|}
\hline & Resultado do & & IIQ & & & & \\
\hline Escore & Set & Mediana & $(25-75 \%)$ & Média & DP & $\mathrm{u}$ & $p$ \\
\hline \multirow[b]{2}{*}{0} & Vencidos & 0 & $2,45-2,73$ & 3,17 & 4,52 & \multirow[b]{2}{*}{495,50} & \multirow[b]{2}{*}{0,807} \\
\hline & Perdidos & 0 & $2,40-2,70$ & 3,07 & 3,76 & & \\
\hline \multirow[b]{2}{*}{1} & Vencidos & 5,55 & $0-6,07$ & 6,73 & 4,75 & \multirow[b]{2}{*}{370,00} & \multirow[b]{2}{*}{0,056} \\
\hline & Perdidos & 9,30 & $0-5,80$ & 9,56 & 6,16 & & \\
\hline \multirow[b]{2}{*}{2} & Vencidos & 13,80 & $8,05-17,98$ & 14,32 & 8,12 & \multirow[b]{2}{*}{469,00} & \multirow[b]{2}{*}{0,563} \\
\hline & Perdidos & 15,22 & $8,90-19,76$ & 15,22 & 8,10 & & \\
\hline \multirow[b]{2}{*}{3} & Vencidos & 78,67 & $70,63-82,13$ & 75,76 & 11,13 & \multirow[b]{2}{*}{414,50} & \multirow[b]{2}{*}{0,190} \\
\hline & Perdidos & 73,50 & $68,50-80,71$ & 72,14 & 11,17 & & \\
\hline
\end{tabular}

$\mathrm{IIQ}=$ Intervalo interquartil. DP $=$ Desvio Padrão. $0=$ erro; $1=$ sem opções de ataque; $2=$ ataque com opções limitadas; e $3=$ todas as opções de ataque.

\section{DISCUSSÃO}

Os principais achados deste estudo revelaram que a qualidade da ação do levantamento foi associada com a zona de distribuição (ofensiva e defensiva). Além disso, foi verificado que os sets vencidos quase não diferem dos perdidos no levantamento, com exceção nas ações que não oferecem opções de ataque, revelando uma forma negativa na organização do ataque dos sets perdidos.

Realizando uma análise com a sequência lógica do jogo, (recepção de saque-levantamento) (PALAO, SANTOS, UREÑA, 2004), percebe-se que é fundamental que a recepção de saque, apesar de não ter sido objeto do presente estudo, seja direcionada para as zonas ofensivas a fim de contribuir na qualidade do levantamento, pois essa ação realizada da zona defensiva pode influenciar negativamente a ação subsequente (GONZÁLEZSILVA et al., 2016). Neste sentido, recepções deficitárias aumentam as chances de derrota no set (COSTA et al., 2014), enquanto que recepções direcionadas para as zonas ofensivas de forma excelente podem aumentar as chances de levantamentos excelentes, além de possibilitar ataques mais rápidos (COSTA et al., 2016).

Ainda na perspectiva da sequência lógica do jogo a qualidade do levantamento também tem papel preponderante para a efetividade do ataque (MATIAS, GRECO, 2011b) que é preditor de sucesso no jogo (MARCELINO, MESQUITA, AFONSO, 2008), sobretudo em categorias femininas no processo de formação (GONZÁLEZSILVA et al., 2016). Assim sendo, é fundamental que treinadores trabalhem com objetividade a qualidade na recepção de saque por meio de diferentes tipos de saques (AFONSO et al., 2012) e que levantadores possam trabalhar com situações de distribuição para as diferentes zonas. Além disso, a maior incidência de bolas levantadas para as posições que compõe à zona ofensiva pode ser justificada em parte devido à confiança do levantador na escolha do atacante por critérios técnicos, táticos (MATIAS, GRECO, 2011c) ou pela própria estrutura organizacional do jogo que predominam ataques nas zonas ofensivas 2, 3 e 4 (INKINEN, HÄYRINEN, LINNAMO, 2013).

Sabe-se que as equipes de alto rendimento utilizam jogadoras com funções específicas (líbero) que qualificam a recepção de 
saque para o levantamento (JOÃO et al., 2006), para facilitar $o$ trabalho $e$ aumentando as possibilidades táticas, que dependerá também de outros fatores para as chances de sucesso como técnica, tática, tomada decisão, conhecimento declarativo e processual para a organização do jogo (MATIAS, GRECO, 2013). No mesmo direcionamento nas categorias de formação o líbero tem importante papel na organização do jogo (FERNANDEZ-ECHEVERRIA, 2015) o que poderá facilitar o levantamento.

Quando comparado o levantamento entre sets vencidos e perdidos, verificou-se que esse resultado não se revelou determinante no sucesso do set. Contudo, os sets perdidos apresentaram mais ações de levantamento que não permitiram a realização de ataques (escore 1). Assim, essa informação é um indicador relevante, pois a não realização de um ataque organizado pode favorecer a formação do contra-ataque da equipe adversária (COSTA et al., 2011). Em um estudo realizado por Costa et al. (2017), foram encontradas diferenças significativas na avaliação da performance no critério continuidade e erro, sendo maior para os sets perdidos, enquanto que os vencidos foram superiores no coeficiente de performance em atletas escolares. Em outro estudo Palao, Santos e Ureña (2004) não encontraram diferenças no levantamento entre os níveis de rendimento das equipes participantes dos Jogos Olímpicos em Sydney 2000. Já Costa et al., (2014) não encontram predição do levantamento com o sucesso do set em atletas femininas adultas participantes da Superliga 2011/2012.

Um ponto que deve ser destacado e que não foi realizado pelo presente estudo foram os levantamentos realizados nos contra-ataques gerados nos complexos 2, 3 e 4 (LAPORTA et al.,
2015). Em um estudo Ramos et al. (2004) encontraram diferenças significativas nos contraataques nas comparações entre equipes participantes do play-off final da Superliga masculina 2002/2003. Assim, este indicador revela a importância que os treinadores devem atribuir ao trabalho com os levantamentos na transição entre os complexos.

Algumas limitações são importantes destacar, não foi levado em consideração o efeito do levantamento na associação com 0 ataque, como a distribuição de bolas para os atacantes em relação aos bloqueadores. Também não foi levada em conta a posição do levantador no rodízio, que influencia diretamente na tática do jogo (PALAO; SANTOS; UREÑA, 2005) e pelo tipo de técnica executada (COSTA, BATISTA, 2015). Futuras pesquisas devem investigar o levantamento dentro do contexto dos complexos e a amplitude de sua influência na finalização do ataque, levando em conta o rodízio imposto pela regra e cortes do jogo.

\section{CONCLUSÃO}

Concluiu-se que a qualidade da ação do levantamento apresentou uma dependência com as zonas ofensivas e defensivas, sendo mais favorável essa associação quando o levantamento foi distribuído para a zona ofensiva. Portanto, levantadores tendem a levantar mais para as zonas ofensivas nesta categoria devido à facilitação da organização do ataque. Em relação às comparações entre sets vencidos e perdidos, conclui-se que os sets vencidos se sobressaem em relação aos perdidos na qualidade do levantamento que não oferecem opções de ataque, o que revela possibilidades de contra-ataque para os sets vencidos.

\section{REFERÊNCIAS}

AFONSO, J. et al. Tactical determinants of setting zone in elite men's volleyball. Journal of Sports Science and Medicine, v. 11, n. 1, p. 64-70, 2012.

COLEMAN, J. Scouting opponents and evaluating team performance. In: SHONDELL, D. (Ed.). The volleyball coaching bible. Champaign: Human Kinetics, 2002. p. 321-346.

COSTA, G. C. et al. Análise das estruturas do complexo I à luz do resultado do set no voleibol feminino. Motricidade, v. 10, n. 3, p. 40-49, 2014.

COSTA, G. T. et al. Análise de jogo no voleibol feminino de formação: análise da associação entre os procedimentos de jogo. Revista Brasileira de Ciência e Movimento, v. 24, n. 4, p. 63-74, 2016.

COSTA, G. C. T. et al. Relação saque, recepção e ataque no voleibol juvenil masculino. Motriz, v. 17, n. 1, p. $11-18,2011$.

COSTA, Y. P.; BATISTA, G. R. Análise da qualidade e desempenho técnico das ações no voleibol feminino escolar. Revista Acta Brasileira do Movimento Humano, v. 5, p. 80-93, 2015.

COSTA Y. P. et al. Indicadores de rendimento técnico-tático em função do resultado do set no voleibol escolar. Motricidade, v. 13, SI, p. 34-40, 2017.

FERNANDEZ-ECHEVERRIA, C. et al. Analysis of the variables that predict serve efficacy in young volleyball players. International Journal of Performance Analysis in Sport, v. 15, n. 1, 2015.

GONZÁLEZ-SILVA, J. et al. Analysis of setting efficacy in young male and female volleyball players. Journal of Human Kinetics, v. 53, n. 1, p. 189-200, 2016. 
HILENO, R.; BUSCÀ, B. Herramienta observacional para analizar la cobertura del ataque en voleibol. Revista Internacional de Medicina y Ciencias de la Actividad Física y el Deporte. v. 12, n. 47, p. 557-570, 2012.

INKINEN, V.; HÄYRINEN, M.; LINNAMO, V. Technical and tactical analysis of women's volleyball. Biomedical Human Kinetics, v. 5, p. 43-50, 2013.

JAMES, N.; TAYLOR, J.; STANLEY, S. Reliability procedures for categorical data in performance analysis. International. Journal of Performance Analysis in Sport, v. 7, n. 1, p. 1-11, 2007.

JOÃO, P. V. et al. Análise comparativa entre o jogador libero e os recebedores prioritários na organização ofensiva , a partir da recepção ao serviço, em voleibol. Revista Portuguesa de Ciências do Desporto, v. 6, n. 3, p. 318-328, 2006.

LANDIS, J. R.; KOCH, G. G. The measurement of observer agreement for categorical data. Biometrics, v. $33, \mathrm{n}$. 1, p. 159-174, 1977.

LAPORTA, L. et al. The Importance of loosely systematized game phases in sports: the case of attack coverage systems in high-level women's. Montenegrin Journal of Sports Science and Medicine, v. 4, n. 1, p. 19-24, 2015.

MAIA, N.; MESQUITA, I. Estudo das zonas e eficácia da recepção em função do jogador recebedor no voleibol sênior feminino. Revista Brasileira de Educação Física e Esporte, v. 20, n. 4, p. 257-270, 2006.

MARCELINO, R. et al. Estudo dos indicadores de rendimento em voleibol em função do resultado do set. Revista Brasileira de Educação Física e Esporte, v. 24, n. 1, p. 69-78, 2010.

MARCELINO, R.; MESQUITA, I.; AFONSO, J. The weight of terminal actions in volleyball contributions of the spike, serve and block for the teams' rankings in the World League 2005. International Journal of Performance Analysis in Sport, v. 8, n. 2, p. 1-7, 2008.

MATIAS, C. J. A. S.; GRECO, P. J. Análise da organização ofensiva dos levantadores campeões da Superliga de voleibol. Revista Brasileira de Ciências do Esporte, v. 33, n. 4, p. 1007-1027, 2011b.

MATIAS, C. J. A. S.; GRECO, P. J. De Morgan ao voleibol moderno: o sucesso do Brasil e a relevância do levantador. Revista Mackenzie de Educação Física e Esporte, v. 10, n. 2, p. 49-63, 2011 a.

MATIAS, C. J. A. S.; GRECO, P. J. Conhecimento tático-estratégico dos levantadores brasileiros campeões de voleibol: da formação ao alto nível. Revista Brasileira de Educação Física e Esporte, v. 25, n. 3, p. 513-535, 2011c.

MATIAS, C. J. A. S.; GRECO, P. J. O conhecimento tático declarativo dos levantadores campeões de voleibol. Motriz, v. 19, n. 1, p. 185-194, 2013.

MILIĆ, M. et al. Anthropometric and physical characteristics allow differentiation of young female volleyball players according to playing position and level of expertise. Biology of Sport, v. 34, n. 1, p. 19-26, 2017.

PALAO, J. M.; SANTOS, J. A.; UREÑA, A. Effect setter's position on the spike in volleyball. Journal of Human Movement Studies, v. 48, n. 1, p. 25-40, 2005.

PALAO, J. M.; MANZANARES, P.; ORTEGA, E. Design, validation, and reliability of an observation instrument for technical and tactical actions in indoor volleyball. European Journal of Human Movement, v. 34, p. 75-95, 2015.

PALAO, J. M.; MANZANARES, P.; ORTEGA, E. Techniques used and efficacy of volleyball skills in relation to gender. International Journal of Performance Analysis in Sport, v. 9, n. 2, p. 281-293, 2009.

PALAO, J. M.; SANTOS, J.; UREÑA, A. Effect of team level on skill performance in volleyball. International Journal of Performance Analysis in Sport, v. 4, n. 2, p. 50-60, 2004.

QUEIROGA, M. et al. O conhecimento tático-estratégico dos levantadores integrantes das seleções brasileiras de voleibol. Fitness \& Performance, v. 9, n. 1, p. 78-92, 2010.

RAMOS, M. H. K. P. et al. Estrutura interna das ações de levantamento das equipes finalistas da superliga masculina de voleibol. Revista Brasileira de Ciência e Movimento, v. 12, n. 4, p. 33-37, 2004.

VALLADARES, N.; GARCÍA-TORMO, J. V.; JOÃO, V. P. Analysis of variables affecting performance in senior female volleyball World Championship 2014. International Journal of Performance Analysis in Sport, v. 16, p. 400-410, 2016. 